\title{
Early time dynamics in heavy-ion collisions from AdS/CFT correspondence
}

\author{
Yuri V. Kovchegov* and Anastasios Taliotis ${ }^{\dagger}$ \\ Department of Physics, The Ohio State University, Columbus, Ohio 43210, USA
}

(Received 29 May 2007; published 26 July 2007)

\begin{abstract}
We study the matter produced in heavy-ion collisions, assuming that this matter is strongly interacting and employing AdS/CFT correspondence to investigate its dynamics. At late proper times $\tau$ we show that Bjorken hydrodynamics solution, obtained recently by Janik and Peschanski using gauge-gravity duality [1], can be singled out by simply requiring that the metric tensor is a real and single-valued function of the coordinates everywhere in the bulk, without imposing any constraints on the curvature invariant. At early proper times we use a similar strategy to show that the energy density $\epsilon$ approaches a constant as $\tau \rightarrow 0$. We therefore demonstrate that the strong coupling dynamics incorporates the isotropization transition in heavy-ion collisions. By matching our early-time regime with the late-time one of Janik and Peschanski we estimate the isotropization time at RHIC to be approximately $\tau_{\text {iso }} \approx 0.3 \mathrm{fm} / c$, in good agreement with results of hydrodynamic simulations.
\end{abstract}

DOI: 10.1103/PhysRevC.76.014905

PACS number(s): $12.38 . \mathrm{Mh}$

\section{INTRODUCTION}

The problem of thermalization of the system produced in heavy-ion collisions is crucial for our understanding of strong interactions. Although in recent years there has been some significant progress in understanding the initial (prethermalization) stages of heavy-ion collisions in the framework of the color glass condensate (CGC) [2-19] (for a review of CGC see Refs. [20-22]), a complete theoretical understanding of the subsequent isotropization and thermalization of the produced medium continues to evade us. The success of hydrodynamics simulation in describing the data generated at the Relativistic Heavy Ion Collider (RHIC) is quite impressive; however, it requires a very early starting proper time for hydrodynamics, $\tau \leqslant 0.5 \mathrm{fm} / c$ [23-30]. The existing theoretical models based on the weak-coupling dynamics so far have not been successful in reproducing such an early thermalization time [31-37]. Moreover, as was suggested by one of the authors [38-40], the perturbative weak-coupling dynamics, describable by Feynman diagrams, may in principle be unable to generate isotropization and, therefore, thermalization in high-energy heavy-ion collisions. It was suggested in Refs. [38-40] that the only way isotropization and thermalization can be achieved in heavy-ion collisions is via strong-coupling effects.

Another more phenomenological argument indicating that strong coupling dynamics could be important for heavy-ion collisions at RHIC is the fact that hydrodynamic description of the collisions [23-30] has to have a very low shear viscosity [41]: the quark-gluon plasma produced at RHIC is almost a perfect fluid. Because the perturbative shear viscosity scales as $\eta \sim 1 / g^{4}$ [42] one concludes that low viscosity requires large coupling. Such conclusion was confirmed by explicit calculations employing gauge-gravity duality in Refs. [43-47].

Unfortunately, the strong coupling regime of quantum chromodynamics (QCD) is not under theoretical control. However, for some gauge theories, like $\mathcal{N}=4$ super YangMills theory, the strong coupling regime can be studied

\footnotetext{
*yuri@mps.ohio-state.edu

†taliotis.1@osu.edu
}

using anti-De-Sitter space/conformal field theory (AdS/CFT) correspondence [48-51]. To understand the strong coupling dynamics of the matter produced in heavy-ion collisions one has to model QCD with $\mathcal{N}=4$ super Yang-Mills theory and use AdS/CFT correspondence to study the strong-coupling dynamics of such theory. In recent years there have been many applications of AdS/CFT correspondence to heavy-ion collisions [1,52-62].

The goal of this article is to attempt to understand the onset of isotropization and thermalization in the AdS/CFT framework. We will consider an idealized Bjorken picture of the collision [63], in which nuclei have an infinite extent in the transverse plane and the distribution of the produced matter is rapidity independent: the energy and pressure components of the energy-momentum tensor in the local rest frame would then depend only on the proper time $\tau$. We emphasize that isotropization and thermalization of the produced medium do not necessarily happen at the same time: isotropization is necessary for thermalization, but not vice versa. As was argued in Ref. [14], in the CGC framework the energy density $\epsilon$ of the matter produced in heavy-ion collisions scales as a power of a logarithm of the proper time $\tau$, as shown in Eq. (53) below. Therefore, the produced matter is anisotropic: its longitudinal pressure component is in fact negative. Isotropization for such matter would imply generation of positive longitudinal pressure, comparable to the transverse pressure components. Note that for isotropization to take place it does not appear necessary for the system to have a thermal distribution: hence thermalization may take place after isotropization and not necessarily at the same time. Below we will study the onset of isotropization, because it is much easier to infer from the components of the energy-momentum tensor.

In a pioneering article [1] Janik and Peschanski showed that for the strongly coupled medium produced in heavy-ion collisions and described by gauge-gravity duality the late time asymptotics can lead only to Bjorken hydrodynamics [63] with the energy-density scaling with proper time as $\epsilon \sim \tau^{-4 / 3}$. Since then a number of further investigations have been carried out $[53,54,64]$, mostly concentrating on the subleading at late times viscous corrections to the ideal hydrodynamic behavior of Ref. [1]. In this work we will take a different route and 
concentrate on the early time behavior of the strongly coupled medium produced in heavy-ion collisions. The hope is to verify if such medium is anisotropic at early proper times: if that proves to be the case then the onset of isotropization would necessarily be a part of strongly coupled medium's dynamics at intermediate proper times. The idea of studying the matter produced in nuclear collisions at small proper times has been originally suggested in the CGC framework in Refs. [14,65]. Here we will apply the same concept to the strong-coupling dynamics employing the AdS/CFT correspondence.

The article is structured as follows. We begin in Sec. II by setting up the formalism of holographic renormalization in the context of heavy-ion collisions with Bjorken geometry. We then proceed in Sec. III by solving Einstein equations (3) in the AdS space assuming that the four-dimensional energy density scales as a power of proper time, as given by Eq. (17). In Sec. III A we will demonstrate the first few steps of the perturbative solution expanding the metric in powers of the Fefferman-Graham coordinate $z$ [66]. In Sec. III B we proceed by reanalyzing the late proper time dynamics: we show that to pick the correct Bjorken hydrodynamic behavior of the solution of Einstein equations (3) one only has to impose the condition that the resulting metric is a real single-valued function of the coordinates everywhere in the bulk. Hence we demonstrate that the condition of nonsingularity of the curvature invariant employed in Ref. [1] is not needed to constrain the dynamics in four dimensions from AdS/CFT. We then apply the same approach to study the early time dynamics in Sec. III C: we solve Einstein equations (3) at small $\tau$ and, after imposing the condition for the metric to be real and single valued, we derive that energy density can only go to a constant value as $\tau \rightarrow 0$ (see Eq. (51)).

This is our main result: as the system having constant energy density in heavy-ion collisions also has negative longitudinal pressure, we conclude that at very early times the strongly interacting system described by gauge-gravity duality is very anisotropic. At the same time we know that at late proper times it becomes isotropic evolving according to Bjorken hydrodynamics. We thus demonstrate that the isotropization transition does take place at intermediate times for the evolution of this strongly-coupled system! Moreover, while indeed the early-time energy density in CGC (Eq. (53)) and in the strongly coupled medium (Eq. (51)) behave somewhat differently, the two initial-time behaviors are not entirely unlike each other: in both cases the initial longitudinal pressure is negative, equal to the negative of the energy density, whereas the transverse pressures are equal to the energy density (see Eq. (52)). We therefore conclude that the behavior of the strongly coupled system studied here may be relevant for real-life heavy-ion collisions, where the initial stages of the collisions are successfully described by the CGC.

We verify our result of constant energy density at early times in Sec. IV, where, inspired by CGC [14], we look for possible logarithmic scaling of the energy density at early time, but find none. The energy density remains constant at small $\tau$, without any logarithmic divergence.

In Sec. $\mathrm{V}$ we match the early time dynamics considered in this work onto the late-time Bjorken hydrodynamic solution found in Ref. [1] to estimate the isotropization time (see
Fig. 1). The result is given by Eq. (81). If we apply this formula to central $\mathrm{Au}+\mathrm{Au}$ collisions at $\mathrm{RHIC}$ at $\sqrt{s}=$ $200 \mathrm{GeV}$ we obtain the isotropization time of $\tau_{\text {iso }} \approx$ $0.3 \mathrm{fm} / c$, in good agreement with results of hydrodynamic simulations.

We conclude in Sec. VI by restating our main results and by summarizing our knowledge of energy density as a function of proper time for the strongly coupled system at hand in Fig. 2.

\section{HOLOGRAPHIC RENORMALIZATION: GENERAL SETUP}

Let us consider a head-on collision of two very large identical nuclei. For simplicity we assume that the distribution of matter produced in such collisions is independent of (space-time) rapidity $\eta$. Because the nuclei are identical the matter distribution should also respect $x_{+} \leftrightarrow x_{-}(\eta \leftrightarrow-\eta)$ symmetry. Finally, as the nuclei are very large, we assume that they have infinite extent in the transverse directions. Therefore the energy-momentum tensor of the produced matter is independent of the transverse coordinates $\underline{x}=\left(x_{1}, x_{2}\right)$. This is indeed the same approximation as employed by Bjorken in Ref. [63].

Following Janik and Peschanski [1] we write the metric in $\mathrm{AdS}_{5}$ space for such a system using Fefferman-Graham coordinates [66] as

$$
\begin{aligned}
d s^{2}= & \frac{1}{z^{2}}\left[-A(\tau, z) d \tau^{2}+\tau^{2} B(\tau, z) d \eta^{2}\right. \\
& \left.+C(\tau, z) d x_{\perp}^{2}+d z^{2}\right],
\end{aligned}
$$

where $\tau=\sqrt{2 x_{+} x_{-}}$is the proper time in four dimensions, $\eta=(1 / 2) \ln \left(x_{+} / x_{-}\right)$is the space-time rapidity, $x_{ \pm}=\left(x_{0} \pm\right.$ $\left.x_{3}\right) / \sqrt{2}, d x_{\perp}^{2}=d x_{1}^{2}+d x_{2}^{2}$ and $z$ is the coordinate in the fifth dimension. Just like in Ref. [1] we write

$A(\tau, z)=e^{a(\tau, z)}, \quad B(\tau, z)=e^{b(\tau, z)}, \quad C(\tau, z)=e^{c(\tau, z)}$,

where $a(\tau, z), b(\tau, z)$, and $c(\tau, z)$ are some unknown functions, to be determined from the solution of Einstein equations with a negative cosmological constant $\Lambda=-6$ in $\mathrm{AdS}_{5}$ space

$$
R_{\mu \nu}-\frac{1}{2} g_{\mu \nu} R-6 g_{\mu \nu}=0 .
$$

Here $R_{\mu \nu}$ is the Ricci tensor and $R$ is the scalar curvature. Contracting Eq. (3) with $g^{\mu v}$ yields

$$
R=-20 \text {, }
$$

which, when inserted back into Eq. (3) gives

$$
R_{\mu \nu}+4 g_{\mu \nu}=0 \text {. }
$$

Finding an exact general solution of Eq. (5) with the metric ansatz (1) appears to be a daunting task. Instead, we begin by exploring the solution using the technique of holographic renormalization [67]. The general prescription for a metric of the form

$$
d s^{2}=\frac{1}{z^{2}}\left[\tilde{g}_{\mu \nu}(x, z) d x^{\mu} d x^{\nu}+d z^{2}\right]
$$

is to expand it near the $z=0$ boundary in a series [67]

$$
\tilde{g}_{\mu \nu}(x, z)=\tilde{g}_{\mu \nu}^{(0)}(x)+z^{2} \tilde{g}_{\mu \nu}^{(2)}(x)+z^{4} \tilde{g}_{\mu \nu}^{(4)}(x)+\cdots
$$

and solve Einstein equations (5) order by order in $z$. 
In our case we know the metric on the $z=0$ boundary: it is simply the flat Minkowski metric, such that $\tilde{g}_{\mu \nu}^{(0)}(x)=\eta_{\mu \nu}$. Then one can show that $\tilde{g}_{\mu \nu}^{(2)}(x)=0$ [67]. Finally, the fourthorder term in Eq. (7) is related to the expectation value of the energy-momentum tensor $T_{\mu \nu}$ in our four dimensions [53,67]

$$
\left\langle T_{\mu \nu}\right\rangle=\frac{N_{c}^{2}}{2 \pi^{2}} \tilde{g}_{\mu \nu}^{(4)}(x)
$$

with $N_{c}$ the number of colors. As can be easily shown [38], the most general energy-momentum tensor in the boost-invariant geometry of the collision of two very large nuclei can be written (in the comoving frame) as

$$
\left\langle T^{\mu \nu}\right\rangle=\left(\begin{array}{cccc}
\epsilon(\tau) & 0 & 0 & 0 \\
0 & p(\tau) & 0 & 0 \\
0 & 0 & p(\tau) & 0 \\
0 & 0 & 0 & p_{3}(\tau)
\end{array}\right)
$$

in the $t, x_{1}, x_{2}, x_{3}$ coordinate system. Here $\epsilon(\tau)$ is the energy density, $p(\tau)$ is the transverse pressure component, and $p_{3}(\tau)$ is the longitudinal pressure component. (The latter two quantities do not have to be equal in general: they are equal only in the case of ideal hydrodynamics.)

In Ref. [1] it was elegantly shown that requiring nonnegativity of the energy density $\epsilon(\tau)$ in all frames leads to the following conditions on its dependence on the proper time $\tau$

$$
\epsilon^{\prime}(\tau) \leqslant 0, \quad \tau \epsilon^{\prime}(\tau) \geqslant-4 \epsilon(\tau) .
$$

We will use this conditions later to restrict energy density.

As will be manifest shortly, Einstein equations lead to the following conditions for the energy-momentum tensor. First, they require energy-momentum conservation in four dimensions

$$
\partial_{\mu}\left\langle T^{\mu \nu}\right\rangle=0
$$

which, when applied to Eq. (9), gives

$$
\frac{d \epsilon}{d \tau}=-\frac{\epsilon+p_{3}}{\tau} .
$$

(Here and below we will sometimes suppress the arguments of $\epsilon, p$, and $p_{3}$.) Second, they require the energy-momentum tensor to be traceless

$$
\left\langle T_{\mu}^{\mu}\right\rangle=0
$$

which, for Eq. (9) leads to

$$
\epsilon=2 p+p_{3} \text {. }
$$

Finally, to apply the expansion of Eq. (7) with $\tilde{g}_{\mu \nu}^{(0)}(x)=\eta_{\mu \nu}$ and $\tilde{g}_{\mu \nu}^{(2)}(x)=0$ to the metric in Eq. (1) we write [1]

$$
\begin{aligned}
& a(\tau, z)=\sum_{n=0}^{\infty} a_{n}(\tau) z^{4+2 n}, \\
& b(\tau, z)=\sum_{n=0}^{\infty} b_{n}(\tau) z^{4+2 n}, \\
& c(\tau, z)=\sum_{n=0}^{\infty} c_{n}(\tau) z^{4+2 n} .
\end{aligned}
$$

Comparing these to Eqs. (8) and (9) yields

$$
\epsilon=-\frac{N_{c}^{2}}{2 \pi^{2}} a_{0}(\tau), \quad p_{3}=\frac{N_{c}^{2}}{2 \pi^{2}} b_{0}(\tau), \quad p=\frac{N_{c}^{2}}{2 \pi^{2}} c_{0}(\tau) .
$$

Now we are ready to implement the holographic renormalization using a particular ansatz for the energy density.

\section{POWER-LAW SCALING OF ENERGY DENSITY WITH PROPER TIME}

\section{A. First steps: iterative solution}

Let us first assume that the energy density scales as

$$
\epsilon(\tau) \sim \tau^{\Delta} \text {. }
$$

This is the simplest assumption. We know that it works at late times $\tau \gg 1$ (in units of $\sqrt{G_{N}}$ with $G_{N}$ the Newton's constant in $\mathrm{AdS}_{5}$ ) [1]. We will apply it to the early-time dynamics $(\tau \ll 1)$ and if it does not work we will be forced to look for a more sophisticated one. At the moment we are not going to make any assumptions or approximations specific to early or late times.

We will begin with the scaling of Eq. (17) and try to build a solution of Einstein equations in the bulk using holographic renormalization. From the dynamics in four dimensions we know that if $\epsilon \sim \tau^{\Delta}$ then $p \sim \tau^{\Delta}$ and $p_{3} \sim \tau^{\Delta}$. Using Eq. (16) we write

$$
a_{0}(\tau)=a_{0} \tau^{\Delta}, b_{0}(\tau)=b_{0} \tau^{\Delta}, c_{0}(\tau)=c_{0} \tau^{\Delta}
$$

with $a_{0}, b_{0}$, and $c_{0}$ some constants. Substituting Eq. (18) into Eq. (15), and using the expansion from the latter in Eq. (5) we solve Einstein equations to the lowest nontrivial order in $z$. This gives

$$
\begin{aligned}
& a_{0}(\tau)=a_{0} \tau^{\Delta} \\
& b_{0}(\tau)=a_{0}(\Delta+1) \tau^{\Delta} \\
& c_{0}(\tau)=-a_{0} \frac{\Delta+2}{2} \tau^{\Delta} .
\end{aligned}
$$

Indeed Eqs. (19) could have been obtained from Eqs. (12) and (14) directly from the four-dimensional considerations of dynamics without trace anomaly. Here we obtain them from the AdS/CFT correspondence.

Substituting Eqs. (19) back into Eq. (15), and again solving Einstein equations (5) at the lowest nontrivial order in $z$ (which is now different from the previous step) yields

$$
\begin{aligned}
& a_{1}(\tau)=a_{0} \frac{\Delta(\Delta+2)}{12} \tau^{\Delta-2} \\
& b_{1}(\tau)=a_{0} \frac{\Delta(\Delta+2)(\Delta-1)}{12} \tau^{\Delta-2} \\
& c_{1}(\tau)=-a_{0} \frac{\Delta^{2}(\Delta+2)}{24} \tau^{\Delta-2} .
\end{aligned}
$$

For what we intend to do next it is instructive to iterate the procedure several more times. Here we will show only the result of the next iteration:

$$
\begin{aligned}
a_{2}(\tau)= & -\frac{1}{384}\left[a_{0} \tau^{\Delta-4}\left(4 \Delta^{2}-\Delta^{4}\right)\right. \\
& \left.+a_{0}^{2} \tau^{2 \Delta} 8\left(8+8 \Delta+3 \Delta^{2}\right)\right]
\end{aligned}
$$




$$
\begin{aligned}
b_{2}(\tau)= & -\frac{1}{384}\left[a_{0} \tau^{\Delta-4}\left(-12 \Delta^{2}+4 \Delta^{3}+3 \Delta^{4}-\Delta^{5}\right)\right. \\
& \left.+a_{0}^{2} \tau^{2 \Delta} 8\left(8+8 \Delta+3 \Delta^{2}\right)\right] \\
c_{2}(\tau)= & -\frac{1}{768}\left[a_{0} \tau^{\Delta-4}\left(8 \Delta^{2}-4 \Delta^{3}-2 \Delta^{4}+\Delta^{5}\right)\right. \\
& \left.+a_{0}^{2} \tau^{2 \Delta} 16\left(8+8 \Delta+3 \Delta^{2}\right)\right] .
\end{aligned}
$$

As follows from the conditions in Eq. (10) [1], positivity of energy density $\epsilon$ combined with the power-law ansatz of Eq. (17) requires that

$$
-4 \leqslant \Delta \leqslant 0 .
$$

Let us assume that $\Delta>-4$. This is a safe assumption: at late times the smallest possible value of $\Delta$ is $\Delta=-4 / 3$ [63]. At late times we therefore have $-4<\Delta \leqslant 0$.

At early times $\Delta \geqslant-1$, the total energy of the matter produced in a collision approximately scales as $E \sim \epsilon \tau$ for small $\tau$. For $\Delta<-1$ the total energy $E$ would be infinite in the $\tau \rightarrow 0$ limit, which is impossible. Hence the physically relevant values of $\Delta$ at early times are constrained to $-1 \leqslant \Delta \leqslant 0$.

\section{B. Rederiving Bjorken hydrodynamics}

Let us first revisit the dynamics at late times, which has already been studied in Ref. [1]. If $\Delta>-4$, then the $\tau^{2 \Delta}$ term dominates over $\tau^{\Delta-4}$ term in Eq. (21), such that Eqs. (19), (20), and (21) combined with Eq. (15) give us the following type of a series for $a(\tau, z)$ in the large- $\tau$ limit

$$
a(\tau, z)=\# z^{4} \tau^{\Delta}+\#^{\prime} z^{8} \tau^{2 \Delta}+\cdots,
$$

with \# and \#' some $\Delta$-dependent constants. (The series for $b(\tau, z)$ and $c(\tau, z)$ are similar.) One can see that $a(\tau, z)$ becomes a function of a single scaling variable $v \propto z \tau^{\Delta / 4}$, as was observed and utilized in Ref. [1].

Following Janik and Peschanski [1] we define the scaling variable as

$$
v=\left(-a_{0}\right)^{1 / 4} z \tau^{\Delta / 4} .
$$

Note that combining Eqs. (16) and (19a) we get

$$
\epsilon(\tau)=-\frac{N_{c}^{2}}{2 \pi^{2}} a_{0} \tau^{\Delta}
$$

such that positivity of the energy density requires that $a_{0}<0$ and the power of $-a_{0}$ in Eq. (24) is well defined. Assuming that $a(\tau, z)=a(v), b(\tau, z)=b(v)$, and $c(\tau, z)=c(v)$ the authors of Ref. [1] solved the Einstein equations (5) for the metric of Eqs. (1) and (2), keeping $v$ constant and taking $\tau \rightarrow \infty$ limit. This led to the following solution obtained in [1]

$$
\begin{aligned}
a(v)= & \frac{1}{2}\left(1-\frac{1}{D}\right) \ln \left(1+D v^{4}\right) \\
& +\frac{1}{2}\left(1+\frac{1}{D}\right) \ln \left(1-D v^{4}\right) \\
b(v)= & \frac{1}{2}\left(1-\frac{\Delta+1}{D}\right) \ln \left(1+D v^{4}\right) \\
& +\frac{1}{2}\left(1+\frac{\Delta+1}{D}\right) \ln \left(1-D v^{4}\right)
\end{aligned}
$$

$$
\begin{aligned}
c(v)= & \frac{1}{2}\left(1+\frac{\Delta+2}{2 D}\right) \ln \left(1+D v^{4}\right) \\
& +\frac{1}{2}\left(1-\frac{\Delta+2}{2 D}\right) \ln \left(1-D v^{4}\right),
\end{aligned}
$$

where

$$
D=\sqrt{\frac{3 \Delta^{2}+8 \Delta+8}{24}} .
$$

To determine the allowed values of the power $\Delta$ let us note that $\ln \left(1-D v^{4}\right)$ has a branch cut along the real axis for $1-$ $D v^{4} \leqslant 0$. Generally speaking this means that $A(\tau, z), B(\tau, z)$, and $C(\tau, z)$ will be complex valued for $1-D v^{4}<0$. Because the $\tau \rightarrow \infty$ limit taken in arriving at Eq. (26) was done keeping $v$ fixed there is no constraint prohibiting $v>D^{-1 / 4}$ and preventing the metric from becoming complex. The only way for the metric to be real and single-valued for all $v$ is if the coefficients in front of $\ln \left(1-D v^{4}\right)$ in Eq. (26) are integers. We therefore require that

$$
\begin{aligned}
\frac{1}{2}\left(1+\frac{1}{D}\right) & =n \\
\frac{1}{2}\left(1+\frac{\Delta+1}{D}\right) & =m \\
\frac{1}{2}\left(1-\frac{\Delta+2}{2 D}\right) & =l
\end{aligned}
$$

with $n, m, l$ some integer numbers. After some straightforward algebra one derives

$$
n+m=2(1-l) .
$$

Therefore $n+m$ is an even number. Then $n-m$ is also an even number. We then write using Eq. (28b)

$$
-\frac{\Delta}{2 D}=n-m=2 k
$$

with $k$ some other integer. Solving

$$
\Delta=-4 D k
$$

for $\Delta$ with the help of Eq. (27) yields

$$
\Delta=-\frac{4}{3} \frac{k}{2 k^{2}-1}\left(2 k \pm \sqrt{3-2 k^{2}}\right) .
$$

As $\Delta \leqslant 0$ and $D \geqslant 0$ we conclude from Eq. (31) that $k \geqslant 0$. For the solutions in Eq. (32) to be real, the only allowed nonnegative values of $k$ are $k=0,1$. If $k=0$ then $\Delta=0$ and $D=1 / \sqrt{3}$. However, then Eq. (28a) will not be satisfied as $n$ will not be integer. If $k=1$ the allowed values of $\Delta$ are $\Delta=-4$ and $\Delta=-\frac{4}{3}$. However, as we concluded from, say, Eq. (21), for $\Delta=-4$ the solution of Eq. (26) is no longer dominant, as terms subleading for larger values of $\Delta$ become comparable to it. On top of that $\Delta=-4$ is not a viable physical value of the power. We are then left only with $\Delta=-\frac{4}{3}$, which, when used in Eq. (25), gives us

$$
\epsilon(\tau)=-\frac{N_{c}^{2}}{2 \pi^{2}} a_{0} \frac{1}{\tau^{4 / 3}} \propto \frac{1}{\tau^{4 / 3}},
$$

characteristic of ideal Bjorken hydrodynamics [63]. We have thus obtained the perfect fluid behavior for the matter produced 
in heavy-ion collisions without imposing a constraint of the absence of singularities in the curvature invariant $\mathcal{R}=$ $R_{\mu \nu \rho \sigma} R^{\mu \nu \rho \sigma}$, like it was done in the original derivation of Ref. [1]. The only necessary requirement was that the metric tensor is a real and single-valued function of $v$ for all values of the scaling variable $v$.

\section{Solution at early times: scaling ansatz}

We will now proceed toward the main goal of this work, which is studying the early-time asymptotics, $\tau \ll 1$. For small $\tau$ the dominant part of the holographic renormalization series studied in Sec. III A is

$$
\begin{aligned}
a(\tau, z) & =\# z^{4} \tau^{\Delta}+\#^{\prime} z^{6} \tau^{\Delta-2}+\#^{\prime \prime} z^{8} \tau^{\Delta-4}+\cdots \\
& =z^{4} \tau^{\Delta}\left(\#+\#^{\prime} \frac{z^{2}}{\tau^{2}}+\#^{\prime \prime} \frac{z^{4}}{\tau^{4}}+\cdots\right),
\end{aligned}
$$

with \#, \#' and \#" some (different) $\Delta$-dependent constants. (The series for $b(\tau, z)$ and for $c(\tau, z)$ have the same structure.) Unfortunately, no single scaling variable can describe the $\tau$ and $z$ dependence now. Nevertheless, as one can see from Eq. (34), the series is in $z / \tau$. Hence, defining a new scaling variable

$$
u \equiv \frac{z}{\tau}
$$

we rewrite Eq. (34) as

$$
a(\tau, u)=\tau^{\Delta+4} u^{4}\left(\#+\#^{\prime} u^{2}+\#^{\prime \prime} u^{4}+\cdots\right) .
$$

Therefore, a general ansatz for $a(\tau, u)$ at early times is

$$
a(\tau, u)=\tau^{\Delta+4} \alpha(u),
$$

where $\alpha(u)$ is some unknown function. Similarly we write

$$
b(\tau, u)=\tau^{\Delta+4} \beta(u), \quad c(\tau, u)=\tau^{\Delta+4} \gamma(u) .
$$

The initial conditions for $\alpha(u), \beta(u)$, and $\gamma(u)$ can be determined from Eqs. (19) yielding

$$
\begin{aligned}
& \alpha(u)=a_{0} u^{4}, \quad \beta(u)=a_{0}(\Delta+1) u^{4}, \\
& \gamma(u)=-a_{0} \frac{\Delta+2}{2} u^{4}, \quad \text { as } \quad u \rightarrow 0 .
\end{aligned}
$$

Our goal now is to use the ansatz of Eqs. (37) and (38) and solve Einstein equations (5) in the $\tau \rightarrow 0$ limit keeping $u$ fixed. In this limit we write

$$
\begin{aligned}
& A(\tau, u)=e^{a(\tau, u)}=e^{\tau^{\Delta+4} \alpha(u)}=1+\tau^{\Delta+4} \alpha(u)+o\left(\tau^{2 \Delta+8}\right) \\
& B(\tau, u)=1+\tau^{\Delta+4} \beta(u)+o\left(\tau^{2 \Delta+8}\right) \\
& C(\tau, u)=1+\tau^{\Delta+4} \gamma(u)+o\left(\tau^{2 \Delta+8}\right)
\end{aligned}
$$

as, with the ansatz of Eqs. (37) and (38), we do not have any control over $o\left(\tau^{2 \Delta+8}\right)$ terms in $A, B$, and $C$.

Rewriting the metric (1) in terms of $\tau, u, \eta$, and $\underline{x}=\left(x_{1}, x_{2}\right)$ we plug it into Eq. (5). At the lowest order in $\tau$ we obtain the following equations, corresponding to the $u u, u \tau$, and $x_{1} x_{1}$ (or, equivalently, $x_{2} x_{2}$ ) components of the Einstein equations:

$$
\frac{\alpha^{\prime}}{2 u}+\frac{\beta^{\prime}}{2 u}+\frac{\gamma^{\prime}}{u}-\frac{\alpha^{\prime \prime}}{2}-\frac{\beta^{\prime \prime}}{2}-\gamma^{\prime \prime}=0,
$$

$$
\begin{aligned}
& \alpha^{\prime}-\frac{3+\Delta}{2} \beta^{\prime}-(2+\Delta) \gamma^{\prime}-\frac{1}{2} u \alpha^{\prime \prime}=0 \\
& \left(8+4 \Delta+\frac{1}{2} \Delta^{2}\right) \gamma+\frac{\alpha^{\prime}}{2 u}+\frac{\beta^{\prime}}{2 u} \\
& +\left(\frac{5}{2 u}-\frac{7}{2} u-\Delta u\right) \gamma^{\prime}+\frac{1}{2}\left(u^{2}-1\right) \gamma^{\prime \prime}=0 .
\end{aligned}
$$

Here $\alpha^{\prime}=d \alpha(u) / d u, \alpha^{\prime \prime}=d^{2} \alpha(u) / d u^{2}$, and the same applies to derivatives of $\beta(u)$ and $\gamma(u)$.

After an integration using the initial conditions (39), Eq. (41) gives

$$
\alpha+\beta+2 \gamma=0 \text {. }
$$

Using Eq. (44) we eliminate $\gamma$ from Eq. (42) to obtain

$$
\beta=(5+\Delta) \alpha-u \alpha^{\prime}
$$

Substituting Eqs. (45) and (44) into Eq. (43) yields

$$
\begin{aligned}
& u^{2}\left(u^{2}-1\right) \alpha^{\prime \prime \prime}+u\left[7+\Delta-(11+3 \Delta) u^{2}\right] \alpha^{\prime \prime} \\
& \quad+\left[-3(5+\Delta)+\left(51+25 \Delta+3 \Delta^{2}\right) u^{2}\right] \alpha^{\prime} \\
& \quad-(4+\Delta)^{2}(6+\Delta) u \alpha=0 .
\end{aligned}
$$

The solution of Eq. (46) satisfying the initial condition (39) can be easily found by searching for it in the form of an infinite series in $u$ starting at the order $u^{4}$. From Eq. (36) we also infer that the coefficient in front of the $u^{5}$ term in the series is zero. The solution of Eq. (46) satisfying such conditions is

$$
\alpha(u)=a_{0} u^{4} F\left(-1-\frac{\Delta}{2},-\frac{\Delta}{2} ; 3 ; u^{2}\right)
$$

with $F$ the hypergeometric function. Equations (45) and (44) allow us to find $\beta(u)$ and $\gamma(u)$. In the end, using Eqs. (40), we obtain the following solution for the components of the metric (1)

$$
\begin{aligned}
A(\tau, u)= & 1+a_{0} \tau^{4+\Delta} u^{4} F\left(-1-\frac{\Delta}{2},-\frac{\Delta}{2} ; 3 ; u^{2}\right), \quad \text { (48a) } \\
B(\tau, u)= & 1+a_{0} \tau^{4+\Delta} u^{4}\left[(\Delta+1) F\left(-1-\frac{\Delta}{2},-\frac{\Delta}{2} ; 3 ; u^{2}\right)\right. \\
& \left.-\frac{\Delta(\Delta+2)}{6} u^{2} F\left(1-\frac{\Delta}{2},-\frac{\Delta}{2} ; 4 ; u^{2}\right)\right], \quad \text { (48b) } \\
C(\tau, u)= & 1+a_{0} \tau^{4+\Delta} u^{4} \frac{\Delta+2}{12} \\
& \times\left[-6 F\left(-1-\frac{\Delta}{2},-\frac{\Delta}{2} ; 3 ; u^{2}\right)\right. \\
& \left.+\Delta u^{2} F\left(1-\frac{\Delta}{2},-\frac{\Delta}{2} ; 4 ; u^{2}\right)\right] .
\end{aligned}
$$

As is well known, hypergeometric functions, such as we have in Eq. (48), have a branch-cut discontinuity along the real axis for $u \geqslant 1$. Therefore, for the metric to be a real and singlevalued function of $u$ at $u>1$ we need the hypergeometric series to terminate. The reasoning here is the same as what we used in rederiving Bjorken hydrodynamics in Sec. III B. As was pointed out at the end of Sec. III A, the allowed values of the power $\Delta$ at early times are $-1 \leqslant \Delta \leqslant 0$. Within this range 
it is possible only for the hypergeometric series to terminate if $\Delta=0$. Therefore, $\Delta=0$ is the only allowed solution.

Plugging in $\Delta=0$ into Eq. (48) gives

$$
\begin{aligned}
& A(\tau, u)=1+a_{0} \tau^{4} u^{4} \\
& B(\tau, u)=1+a_{0} \tau^{4} u^{4} \\
& C(\tau, u)=1-a_{0} \tau^{4} u^{4} .
\end{aligned}
$$

Using $\Delta=0$ in Eqs. (19) and (16) yields

$$
\epsilon=-\frac{N_{c}^{2}}{2 \pi^{2}} a_{0}, \quad p_{3}=\frac{N_{c}^{2}}{2 \pi^{2}} a_{0}, \quad p=-\frac{N_{c}^{2}}{2 \pi^{2}} a_{0} .
$$

Note that at early times $a_{0}$ is different from the one used at late times in Sec. III B. Still $a_{0}<0$ and we conclude from Eq. (50) that, for the strongly coupled medium described by gauge-gravity duality in the geometry of heavy-ion collisions

$$
\epsilon(\tau) \rightarrow \text { constant as } \tau \rightarrow 0 \text {. }
$$

We also obtain that, as $\tau \rightarrow 0$

$$
p_{3}(\tau)=-\epsilon(\tau), \quad p(\tau)=\epsilon(\tau) .
$$

The latter relations one can also obtain from Eqs. (12) and (14) by using Eq. (51) in them.

Note that for the description of heavy-ion collisions in the framework of the CGC, in which the produced medium has a small coupling constant, it was suggested recently by Lappi [14] (see also Ref. [68]) that the leading behavior of energy density as $\tau \rightarrow 0$ is given by

$$
\epsilon_{\mathrm{CGC}}(\tau) \propto \ln ^{2}\left(\frac{1}{\tau}\right) \quad \text { as } \quad \tau \rightarrow 0 .
$$

Using Eq. (53) in Eqs. (12) and (14) and keeping leading logarithms only one arrives at Eq. (52). Hence, although Eq. (53) is indeed different from our Eq. (51), the energy density and pressures at early proper times behave somewhat similar for the cases of CGC and the strongly coupled medium considered above. In both cases the initial longitudinal pressure is negative, whereas the transverse pressure is positive and equal to the energy density.

\section{LOGARITHMIC SCALING OF ENERGY DENSITY WITH PROPER TIME}

In the previous section we looked for the energy density at early proper times using the power-law ansatz (17) and found that the power of $\tau$ has to be zero. However, in view of the CGC result (53) [14] it may be that the absence of a power-law scaling indicates some residual logarithmic scaling of energy density with proper time. Here we will verify whether such logarithmic scaling does indeed take place.

It is tempting to begin by assuming that the energy density scales logarithmically with $\tau$ such that

$$
\epsilon(\tau) \propto \ln ^{\delta}\left(\frac{1}{\tau}\right)
$$

with some number $\delta \geqslant 0$. However, if we try solving Einstein equations (5) with the ansatz (54) at $z=0$ keeping the leading logarithmic $(\ln (1 / \tau))$ terms at small $\tau$ we will simply reproduce the results of the previous section (with the second terms in each of the equations (49) multiplied by $\left.\ln ^{\delta}\left(\frac{1}{\tau}\right)\right)$ without obtaining any constraints on $\delta$. The reason for that is quite straightforward: if one differentiates a power of $\ln (1 / \tau)$ with respect to $\tau$ one power of the logarithm would be lost and such contribution would not be leading logarithmic anymore and would have to be discarded. Therefore, in the leading logarithmic approximation no derivative acts on the power of the logarithm, making $\ln ^{\delta}\left(\frac{1}{\tau}\right)$ term just an overall factor canceling out in the Einstein equations and not giving any constraint on $\delta$.

To avoid this problem one has to include the subleading logarithmic correction. We start by writing

$$
a_{0}(\tau)=a_{0} \ln ^{\delta}\left(\frac{1}{\tau}\right)+a_{1} \ln ^{\delta-1}\left(\frac{1}{\tau}\right)+\cdots
$$

such that, using Eq. (16),

$$
\epsilon(\tau)=-\frac{N_{c}^{2}}{2 \pi^{2}}\left[a_{0} \ln ^{\delta}\left(\frac{1}{\tau}\right)+a_{1} \ln ^{\delta-1}\left(\frac{1}{\tau}\right)+\cdots\right] .
$$

Here $a_{1}$ is some undetermined constant. The ellipsis denote terms of the order $o\left[\ln ^{\delta-2}\left(\frac{1}{\tau}\right)\right]$. Using Eq. (56) in Eqs. (12) and (14) yields

$$
\begin{aligned}
& b_{0}(\tau)=a_{0} \ln ^{\delta}\left(\frac{1}{\tau}\right)+\left(a_{1}-a_{0} \delta\right) \ln ^{\delta-1}\left(\frac{1}{\tau}\right)+\cdots \\
& c_{0}(\tau)=-a_{0} \ln ^{\delta}\left(\frac{1}{\tau}\right)+\left(\frac{a_{0} \delta}{2}-a_{1}\right) \ln ^{\delta-1}\left(\frac{1}{\tau}\right)+\cdots
\end{aligned}
$$

We performed a perturbative expansion of the solution of Einstein equations, similar to the one carried out in Sec. III A. It indicated that the scaling variable is still $u$ defined in Eq. (35). Therefore we try looking for the solution of Eq. (5) in the following form

$$
\begin{aligned}
& A(\tau, u)=1+a_{0} \tau^{4} u^{4} \ln ^{\delta}\left(\frac{1}{\tau}\right)+\tau^{4} \alpha_{1}(u) \ln ^{\delta-1}\left(\frac{1}{\tau}\right)+\cdots \\
& B(\tau, u)=1+a_{0} \tau^{4} u^{4} \ln ^{\delta}\left(\frac{1}{\tau}\right)+\tau^{4} \beta_{1}(u) \ln ^{\delta-1}\left(\frac{1}{\tau}\right)+\cdots \\
& C(\tau, u)=1-a_{0} \tau^{4} u^{4} \ln ^{\delta}\left(\frac{1}{\tau}\right)+\tau^{4} \gamma_{1}(u) \ln ^{\delta-1}\left(\frac{1}{\tau}\right)+\cdots
\end{aligned}
$$

with the unknown functions $\alpha_{1}(u), \beta_{1}(u)$, and $\gamma_{1}(u)$ satisfying the initial conditions which follow from Eqs. (55), (57)

$$
\begin{aligned}
& \alpha_{1}(u)=a_{1} u^{4}, \quad \beta_{1}(u)=\left(a_{1}-a_{0} \delta\right) u^{4}, \\
& \gamma_{1}(u)=\left(\frac{a_{0} \delta}{2}-a_{1}\right) u^{4}, \quad \text { as } \quad u \rightarrow 0 .
\end{aligned}
$$

The ansatz of Eq. (58) trivially satisfies the Einstein equations at the leading logarithmic order for the reasons discussed above. Nontrivial information comes in at the subleading logarithmic order. At the order $\ln ^{\delta-1}\left(\frac{1}{\tau}\right)$ the $u u, x_{1} x_{1}$, and $u x_{1}$ 
components of the Einstein equations (5) read

$$
\begin{aligned}
\frac{\alpha_{1}^{\prime}}{2 u}+\frac{\beta_{1}^{\prime}}{2 u}+\frac{\gamma_{1}^{\prime}}{u}-\frac{\alpha_{1}^{\prime \prime}}{2}-\frac{\beta_{1}^{\prime \prime}}{2}-\gamma_{1}^{\prime \prime} & =0, \\
16 u \gamma_{1}+\alpha_{1}^{\prime}+\beta_{1}^{\prime}+\left(5-7 u^{2}\right) \gamma_{1}^{\prime}+u\left(u^{2}-1\right) \gamma_{1}^{\prime \prime} & =0, \\
4 a_{0} \delta u^{3}-2 \alpha_{1}^{\prime}+3 \beta_{1}^{\prime}+4 \gamma_{1}^{\prime}+u \alpha_{1}^{\prime \prime} & =0 .
\end{aligned}
$$

Just like in Sec. III C we integrate Eq. (60) to obtain

$$
\alpha_{1}+\beta_{1}+2 \gamma_{1}=0 \text {. }
$$

Using Eq. (63) in Eq. (61) to eliminate $\alpha_{1}^{\prime}+\beta_{1}^{\prime}$ yields

$$
16 u \gamma_{1}+\left(3-7 u^{2}\right) \gamma_{1}^{\prime}+u\left(u^{2}-1\right) \gamma_{1}^{\prime \prime}=0 .
$$

The only solution of Eq. (64) satisfying the initial conditions (59) is

$$
\gamma_{1}(u)=\left(\frac{a_{0} \delta}{2}-a_{1}\right) u^{4} .
$$

Using the solution (65) in Eqs. (62) and (63) yields

$$
u \alpha_{1}^{\prime \prime}-5 \alpha_{1}^{\prime}+8 a_{1} u^{3}=0 .
$$

The solution of Eq. (66) satisfying the initial conditions (59) is

$$
\alpha_{1}(u)=a_{1} u^{4} .
$$

Finally, plugging Eqs. (65) and (67) into Eq. (63) yields

$$
\beta_{1}(u)=\left(a_{1}-a_{0} \delta\right) u^{4} .
$$

To make sure that Eqs. (67), (68), and (65) give us the solution of Eq. (5) we need to verify that the remaining two equations corresponding to $\eta \eta$ and $\tau \tau$ components of Eq. (5) are satisfied. These read

$$
\begin{aligned}
& 2 a_{0} \delta u^{5}-4 u \alpha_{1}+20 u \beta_{1}+8 u \gamma_{1}+\left(1+u^{2}\right) \alpha_{1}^{\prime} \\
& \quad+4\left(1-2 u^{2}\right) \beta_{1}^{\prime}+2\left(1-u^{2}\right) \gamma_{1}^{\prime}-u\left(1-u^{2}\right) \beta_{1}^{\prime \prime}=0
\end{aligned}
$$

and

$$
\begin{aligned}
& -6 a_{0} \delta u^{5}+4 u \alpha_{1}-20 u \beta_{1}-24 u \gamma_{1}+2\left(u^{2}-2\right) \alpha_{1}^{\prime} \\
& -\left(u^{2}-1\right)\left(-\beta_{1}^{\prime}-2 \gamma_{1}^{\prime}+u \alpha_{1}^{\prime \prime}\right)=0 .
\end{aligned}
$$

Substituting Eqs. (67), (68), and (65) into Eqs. (69) and (70) we see that the latter equations can be satisfied for $a_{0} \neq 0$ only if

$$
\delta=0 \text {. }
$$

This constrains the power of the logarithm in Eq. (54). Putting $\delta=0$ in Eq. (56) we obtain

$$
\epsilon(\tau)=-\frac{N_{c}^{2}}{2 \pi^{2}}\left[a_{0}+\frac{a_{1}}{\ln (1 / \tau)}+\cdots\right], \quad \text { as } \quad \tau \rightarrow 0 .
$$

We therefore confirm the result of the previous section that the energy density approaches a constant as $\tau \rightarrow 0$. Equation (72) demonstrates that the approach to the constant may be logarithmic.

Again our derivation never employed the requirement of no singularities of the curvature invariant $\mathcal{R}=R_{\mu \nu \rho \sigma} R^{\mu \nu \rho \sigma}$ of [1]. At the same time it can be explicitly checked that it does not violate such a requirement. The curvature invariant for the solution in Eq. (49) (which is also the leading small- $\tau$ solution for the logarithmic scaling considered in this section, as follows from the above) is

$$
\mathcal{R}=40+o\left(\tau^{8}\right) .
$$

Here we keep $u$ fixed and take $\tau$ to be small. Equation (73) shows that the nontrivial part of the solution (49) does not contribute to the curvature invariant and, therefore, does not introduce singularities in it. In fact one can show in general that the first perturbative correction to the metric of the flat AdS space satisfying Eq. (5) does not contribute to the curvature invariant. It appears that it would have been impossible to constrain the early times scaling of the energy density using nonsingularity of the curvature invariant only. Luckily, we were able to pinpoint the right metric without imposing any additional conditions.

\section{ESTIMATE OF ISOTROPIZATION TIME}

We have shown above that the strongly coupled system produced in heavy-ion collisions with the dynamics described by the AdS/CFT correspondence starts off at early times with a finite energy density and negative longitudinal pressure component of the energy-momentum tensor. Hence, the system at these early times is very asymmetric. However, as was demonstrated by Janik and Peschanski [1], the same strongly coupled system at asymptotically late times behaves like the ideal Bjorken hydrodynamics, with the longitudinal and transverse pressure components equal and positive. Therefore, the dynamics of this strongly coupled system incorporates the isotropization transition at some intermediate time and has the potential of solving the thermalization problem in heavy-ion collisions. Indeed, because the initial condition in real heavy ion collisions described by QCD are likely to be perturbative describable by CGC, to verify the relevance of the strongly-coupled model considered above to the real-life heavy-ion collisions it is important to check whether the early times dynamics obtained here agrees with perturbative expectations. The very early time dynamics was studied in the CGC framework in Ref. [14] with the conclusion that the energy-density scales as shown in Eq. (53). Such behavior also leads to negative initial longitudinal pressure, similar to what we observed for the strongly coupled theory. Therefore it is plausible that the strongly coupled isotropization scenario is relevant for the real-life heavy-ion collisions described by QCD.

With that in mind let us estimate the onset of isotropic Bjorken hydrodynamics in the gauge-gravity duality framework considered here. First, at early times the general solution (48) has a branch-cut discontinuity at $u \geqslant 1$. In the $z, \tau$ space the branch cut is at

$$
z \geqslant z_{c}^{\text {early }} \equiv \tau
$$

However, the solution found by Janik and Peschanski [1] and shown here in Eq. (26) has a branch cut at

$$
v \geqslant D^{-\frac{1}{4}}
$$


which, with the help of Eq. (24) with $\Delta=-4 / 3$ and corresponding $D=1 / 3$, translates into

$$
z \geqslant z_{c}^{\text {late }} \equiv \tau^{\frac{1}{3}}\left(\frac{3}{-a_{0}}\right)^{\frac{1}{4}}
$$

The locations of the starting points of the branch cuts $z_{c}^{\text {early }}$ and $z_{c}^{\text {late }}$ given by Eqs. (74) and (76) are shown in the $z, \tau$ plane in Fig. 1. The two curves in Fig. 1 intercept at some time which we label $\tau_{\text {iso }}$.

It is important to point out that, as can be seen, say, from Eq. (21), the full solution with the power-law ansatz of Eq. (17) consists of a sum (for $a, b$, and $c$ ) of the solution in Eq. (26) [1] and the solution in Eq. (48) found above. Although different solutions dominate at different times, both exist at all times. We now observe that to pinpoint the correct solution using the power-law ansatz of Eq. (17) as was done above one starts outside the branch cut, i.e., at $z<z_{c}$ for both early and late times, and imposes the no-branch-cut condition as $z$ increases past $z_{c}$. For the times before the intersection of the two curves in Fig. $1, \tau<\tau_{\text {iso }}$, the first branch cut which has to be eliminated is the one starting at $z_{c}^{\text {early }}$. That fixes the power $\Delta=0$ making the energy density a constant, as described above and shown in Eq. (51). At late times the first branch cut to be eliminated is at $z_{c}^{\text {late }}$, fixing $\Delta=-4 / 3$ as described in Sec. III B. Hence the behavior of energy density, or, equivalently, the dominance of either one of the solutions in Eqs. (26) and (48), is determined by which one of the two branch cuts starts at smaller values of $z$. At $z_{c}^{\text {early }}<z_{c}^{\text {late }}$ the early-time solution dominates leading to the energy-density scaling of Eq. (51). At $z_{c}^{\text {early }}>z_{c}^{\text {late }}$ the late-time solution dominates leading to Bjorken's energy density scaling of Eq. (33). Although, strictly speaking both solutions are valid only at either asymptotically early or late times far away from the

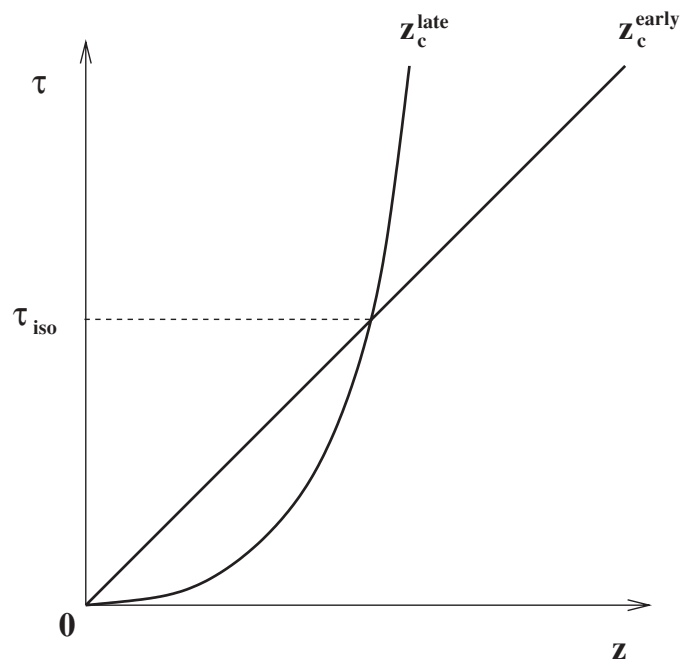

FIG. 1. The locations of branching points $z_{c}^{\text {early }}$ and $z_{c}^{\text {late }}$ for the early- and late-time metrics in the $z, \tau$ plane. The intersection of the two curves gives our estimate of the isotropization time $\tau_{\text {iso }}$ (see text). intersection point shown in Fig. 1, we can estimate the time of the transition between the two regimes by the time of the intercept $\tau_{\text {iso }}$, defined by the condition

$$
z_{c}^{\text {early }}=z_{c}^{\text {late }} .
$$

Using Eqs. (74) and (76) in Eq. (77) yields

$$
\tau_{\text {iso }}=\left(\frac{3}{-a_{0}}\right)^{\frac{3}{8}} \text {. }
$$

This is our estimate of the isotropization time, the time necessary for hydrodynamics to work and required for thermalization. To determine $a_{0}$ we write Bjorken energy-density scaling as

$$
\epsilon(\tau)=\frac{\epsilon_{0}}{\tau^{\frac{4}{3}}} .
$$

Comparing Eqs. (79) and (33) we see that

$$
a_{0}=-\frac{2 \pi^{2}}{N_{c}^{2}} \epsilon_{0}
$$

which, when substituted into Eq. (78), gives

$$
\tau_{\text {iso }}=\left(\frac{3}{\epsilon_{0}} \frac{N_{c}^{2}}{2 \pi^{2}}\right)^{\frac{3}{8}} .
$$

Note that for a self-consistent conformally invariant strongly interacting theory the above result is natural: from the scaling of Eq. (79) we see that such theory would be characterized only by one dimensionful parameter- $\epsilon_{0}$. Using this parameter we can construct the time scale, which is $\epsilon_{0}^{-3 / 8}$. Indeed to find the coefficient in front of $\epsilon_{0}^{-3 / 8}$ in Eq. (81) one needs to perform a more complete calculation, as was done above.

To evaluate the isotropization time in Eq. (81) for central $\mathrm{Au}+\mathrm{Au}$ collisions with $\sqrt{s}=200 \mathrm{GeV}$ at RHIC we use the fact that hydrodynamic simulations of [23-27], which successfully describe a variety of RHIC observables, yield the averaged over all impact parameters energy density of $\epsilon=15$ $\mathrm{GeV} / \mathrm{fm}^{3}[27,69]$ at the proper time $\tau=0.6 \mathrm{fm} / c$. From these data, using Eq. (79) we obtain $\epsilon_{0} \approx 38 \mathrm{fm}^{-8 / 3}$. Substituting this number into Eq. (81) with $N_{c}=3$ yields

$$
\tau_{\text {iso }} \approx 0.29 \mathrm{fm} / c,
$$

which is quite close to the initialization time for the hydrodynamic simulations required to describe RHIC data [23-30,69].

Indeed the isotropization time estimate of Eq. (81) depends on the final state observable $\epsilon_{0}$ instead of some initialstate observable. Therefore one should indeed question the predictive power of such estimate. We leave it for the future work to improve on this result and to tie it to some initial state observables.

\section{CONCLUSIONS}

We conclude by restating our main results. We have rederived the result of Janik and Peschanski [1] stating that at late proper times the system produced in heavy-ion collisions in the strongly coupled regime described by gauge-gravity duality 


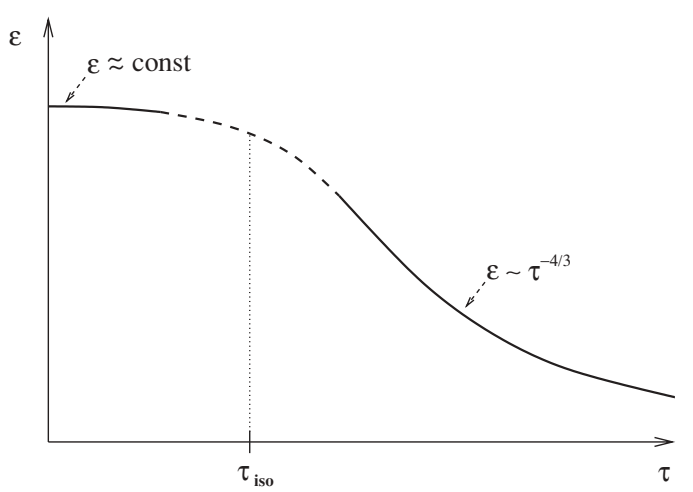

FIG. 2. The scaling of energy density $\epsilon$ with proper time $\tau$ in heavy-ion collisions for a strongly coupled system as described by AdS/CFT correspondence. At late time Bjorken hydrodynamics is recovered, as was shown in Ref. [1]. At early times the energy density goes to a constant, as shown in this work. Our estimate of the isotropization time $\tau_{\text {iso }}$ is likely located in some transition (isotropization) region.

exhibits energy-density scaling with time characteristic of Bjorken hydrodynamics, as shown in Eq. (33). In such a regime all three pressure components of the energy-momentum tensor are equal to each other, as is required for a thermalized medium. To obtain this result we use a much weaker condition than that used in Ref. [1]: we require the metric to be a real and single-valued function of coordinates in the bulk.

We then explore the dynamics of the same strongly coupled system at early times and show that the energy density approaches a constant as $\tau \rightarrow 0$, as shown in Eq. (51). The longitudinal pressure of this early system is therefore negative [see Eq. (12)]. We have therefore demonstrated that this strongly coupled system is initially anisotropic, but at late times it becomes isotropic and evolves according to Bjorken hydrodynamics. Therefore, a complete solution for the energy density of this system at all times would contain the isotropization transition. The similarity of the early-time behavior of the system to that of the CGC demonstrates that our conclusions may be relevant to heavy-ion collisions in the real world.

Figure 2 summarizes our knowledge of the energy density in heavy-ion collisions as a function of $\tau$ for the strongly coupled system considered above and in Ref. [1]. The energy density starts out as a constant, after which the system undergoes an isotropization transition (denoted by the dashed line in Fig. 2) resulting in Bjorken hydrodynamics. The exact shape of the dashed curve is indeed unknown: what we plot in Fig. 2 is a simple interpolation between the early- and late-time regimes. More work is needed to develop a better understanding of this important intermediate region.

Finally, above we estimated the time of the transition from our regime to that of Janik and Peschanski. The estimate is given in Eq. (81). If applied to RHIC, it appears to give the isotropization time $\tau_{\text {iso }} \approx 0.3 \mathrm{fm} / c$, consistent with the results of hydrodynamic simulations [23-30].

In conclusion let us point out that the method of requiring the metric to be a real and single-valued function of coordinates could be successfully applied to other related problems. Recently $o\left(1 / \tau^{2 / 3}\right)$ and $o\left(1 / \tau^{4 / 3}\right)$ corrections to the solution of Eq. (26) have been calculated in Refs. [53,54] for late proper times. One expands $[53,54]$

$$
a(\tau, z)=a(v)+a_{1}(v) \frac{1}{\tau^{2 / 3}}+a_{2}(v) \frac{1}{\tau^{4 / 3}}+\cdots
$$

and finds the subleading coefficients $a_{1}(v)$ and $a_{2}(v)$ by solving Einstein equations perturbatively. [The same expansion is done for $b(\tau, z)$ and $c(\tau, z)$.] Taking the $o\left(1 / \tau^{4 / 3}\right)$ correction to the coefficient $a(v)$ in Eq. (26), denoted by $a_{2}(v)$ and given in Eq. (20) of [53], one can see that it also has a term proportional to $\ln \left(1-v^{4} / 3\right)$, just like the solution in Eq. (26) with $\Delta=$ $-4 / 3$ :

$$
\begin{aligned}
a_{2}(v)= & \left(\frac{1}{4 \sqrt{3}}-\frac{3 \eta_{0}^{2}}{2}\right) \ln \left(1-D v^{4}\right) \\
& + \text { terms without branch cuts. }
\end{aligned}
$$

Here $\eta_{0}$ is the proper-time independent coefficient related to shear viscosity $\eta$ by $[53,54]$

$$
\eta=\frac{\eta_{0}}{\tau} .
$$

Again we want the metric to be real and single-valued, hence the coefficient in front of $\ln \left(1-D v^{4}\right)$ has to be an integer. Note, however, that $a_{2}(v)$ enters the metric with a prefactor of $1 / \tau^{4 / 3}$ as can be seen from Eq. (83), such that the coefficient in front of the logarithm in the metric varies with time. Therefore we conclude that the coefficient in front of $\ln \left(1-D v^{4}\right)$ can only be zero: this would ensure that the metric is real at all times. Putting the coefficient in front of $\ln \left(1-D v^{4}\right)$ in Eq. (84) to zero yields

$$
\eta_{0}^{2}=\frac{\sqrt{3}}{18}
$$

in agreement with the result obtained in Ref. [53] using nonsingularity of the curvature invariant criterion and, as shown in Ref. [53], in agreement with the Kovtun-PolicastroSon-Starinets (KPSS) viscosity bound [43-47].

Here we have to caution the reader that similar branch cuts in other metric coefficients, like $b_{1}(v), b_{2}(v)$, and $c_{1}(v), c_{2}(v)$ of Ref. [53] cannot be eliminated this easily. $\left(a_{1}(v)\right.$ has no branch cut singularity [53].) It is possible that resummation of the whole series of the type shown in Eq. (83) for $b(\tau, z)$ and $c(\tau, z)$ would be required to eliminate such branch cuts. We leave the investigation of this important question for future work.

\section{ACKNOWLEDGMENTS}

We thank Ulrich Heinz, Romuald Janik, Robi Peschanski, Dam Son, and Heribert Weigert for stimulating and informative discussions. This research is sponsored in part by the U.S. Department of Energy under grant DE-FG02-05ER41377. 
[1] R. A. Janik and R. Peschanski, Phys. Rev. D 73, 045013 (2006).

[2] J. P. Blaizot and A. H. Mueller, Nucl. Phys. B289, 847 (1987).

[3] L. McLerran and R. Venugopalan, Phys. Rev. D 49, 2233 (1994).

[4] L. McLerran and R. Venugopalan, Phys. Rev. D 49, 3352 (1994).

[5] L. D. McLerran and R. Venugopalan, Phys. Rev. D 50, 2225 (1994).

[6] Y. V. Kovchegov, Phys. Rev. D 54, 5463 (1996).

[7] Y. V. Kovchegov, Phys. Rev. D 55, 5445 (1997).

[8] A. Kovner, L. D. McLerran, and H. Weigert, Phys. Rev. D 52, 6231 (1995).

[9] Y. V. Kovchegov and D. H. Rischke, Phys. Rev. C 56, 1084 (1997).

[10] A. Krasnitz and R. Venugopalan, Nucl. Phys. B557, 237 (1999).

[11] A. Krasnitz and R. Venugopalan, Phys. Rev. Lett. 84, 4309 (2000).

[12] A. Krasnitz, Y. Nara, and R. Venugopalan, Nucl. Phys. A727, 427 (2003).

[13] Y. V. Kovchegov, Nucl. Phys. A692, 557 (2001).

[14] T. Lappi, Phys. Rev. C 67, 054903 (2003).

[15] D. Kharzeev and M. Nardi, Phys. Lett. B507, 121 (2001).

[16] D. Kharzeev, E. Levin, and M. Nardi, Phys. Rev. C 71, 054903 (2005).

[17] D. Kharzeev, E. Levin, and L. McLerran, Phys. Lett. B561, 93 (2003).

[18] D. Kharzeev, Y. V. Kovchegov, and K. Tuchin, Phys. Lett. B599, 23 (2004).

[19] J. L. Albacete, N. Armesto, A. Kovner, C. A. Salgado, and U. A. Wiedemann, Phys. Rev. Lett. 92, 082001 (2004).

[20] E. Iancu and R. Venugopalan, Invited Review for 'Quark Gluon Plasma 3', edited by R. Hwa and X. N. Wang (World Scientific, Singapore), p. 249.

[21] H. Weigert, Prog. Part. Nucl. Phys. 55461 (2005).

[22] J. Jalilian-Marian and Y. V. Kovchegov, Prog. Part. Nucl. Phys. 56, 104 (2006).

[23] P. F. Kolb, J. Sollfrank, and U. Heinz, Phys. Rev. C 62, 054909 (2000).

[24] P. F. Kolb, P. Huovinen, U. W. Heinz, and H. Heiselberg, Phys. Lett. B500, 232 (2001).

[25] P. Huovinen, P. F. Kolb, U. W. Heinz, P. V. Ruuskanen, and S. A. Voloshin, Phys. Lett. B503, 58 (2001).

[26] P. F. Kolb, U. W. Heinz, P. Huovinen, K. J. Eskola, and K. Tuominen, Nucl. Phys. A696, 197 (2001).

[27] U. W. Heinz and P. F. Kolb, Nucl. Phys. A702, 269 (2002).

[28] D. Teaney and E. V. Shuryak, Phys. Rev. Lett. 83, 4951 (1999).

[29] D. Teaney, J. Lauret, and E. V. Shuryak, Phys. Rev. Lett. 86, 4783 (2001).

[30] D. Teaney, J. Lauret, and E. V. Shuryak, http://xxx. lanl.gov/abs/nucl-th/0110037 nucl-th/0110037.

[31] R. Baier, A. H. Mueller, D. Schiff, and D. T. Son, Phys. Lett. B502, 51 (2001).

[32] S. Mrowczynski, Phys. Lett. B214, 587 (1988).

[33] S. Mrowczynski, Phys. Lett. B314, 118 (1993).

[34] P. Arnold, J. Lenaghan, and G. D. Moore, J. High Energy Phys. 08 (2003) 002.

[35] P. Arnold, J. Lenaghan, G. D. Moore, and L. G. Yaffe, Phys. Rev. Lett. 94, 072302 (2005).
[36] A. Rebhan, P. Romatschke, and M. Strickland, Phys. Rev. Lett. 94, 102303 (2005).

[37] P. Romatschke and R. Venugopalan, Phys. Rev. D 74, 045011 (2006).

[38] Y. V. Kovchegov, Nucl. Phys. A762, 298 (2005).

[39] Y. V. Kovchegov, Nucl. Phys. A764, 476 (2006).

[40] Y. V. Kovchegov, Nucl. Phys. A774, 869 (2006).

[41] D. Teaney, Phys. Rev. C 68, 034913 (2003).

[42] P. Arnold, G. D. Moore, and L. G. Yaffe, J. High Energy Phys. 11 (2000) 001.

[43] G. Policastro, D. T. Son, and A. O. Starinets, Phys. Rev. Lett. 87, 081601 (2001).

[44] D. T. Son and A. O. Starinets, J. High Energy Phys. 09 (2002) 042.

[45] G. Policastro, D. T. Son, and A. O. Starinets, J. High Energy Phys. 09 (2002) 043.

[46] P. Kovtun, D. T. Son, and A. O. Starinets, J. High Energy Phys. 10 (2003) 064.

[47] P. Kovtun, D. T. Son, and A. O. Starinets, Phys. Rev. Lett. 94, 111601 (2005).

[48] J. M. Maldacena, Adv. Theor. Math. Phys. 2, 231 (1998); Int. J. Theor. Phys. 38, 1113 (1999).

[49] S. S. Gubser, I. R. Klebanov, and A. M. Polyakov, Phys. Lett. B428, 105 (1998).

[50] E. Witten, Adv. Theor. Math. Phys. 2, 253 (1998).

[51] O. Aharony, S. S. Gubser, J. M. Maldacena, H. Ooguri, and Y. Oz, Phys. Rep. 323, 183 (2000).

[52] R. A. Janik and R. Peschanski, Phys. Rev. D 74, 046007 (2006).

[53] R. A. Janik, Phys. Rev. Lett. 98, 022302 (2007).

[54] S. Nakamura and S.-J. Sin, J. High Energy Phys. 09 (2006) 020.

[55] E. Shuryak, S.-J. Sin, and I. Zahed, J. Korean Phys. Soc. 50, 384 (2007).

[56] S. Lin and E. Shuryak, http://xxx.lanl.gov/abs/hep-ph/0610168 hep-ph/0610168.

[57] M. Lublinsky and E. Shuryak, http://xxx.lanl.gov/abs/arXiv: 0704.1647 [hep-ph] arXiv:0704.1647 [hep-ph].

[58] H. Nastase, http://xxx.lanl.gov/abs/hep-th/0501068 hep-th/ 0501068.

[59] D. Bak and R. A. Janik, Phys. Lett. B645, 303 (2007).

[60] K. Kajantie, T. Tahkokallio, and J.-T. Yee, J. High Energy Phys. 01 (2007) 019.

[61] K. Kajantie and T. Tahkokallio, Phys. Rev. D 75, 066003 (2007).

[62] K. Kajantie, J. Louko, and T. Tahkokallio, http://xxx.lanl. gov/abs/arXiv:0705.1791 [hep-th] arXiv:0705.1791 [hep-th].

[63] J. D. Bjorken, Phys. Rev. D 27, 140 (1983).

[64] M. P. Heller and R. A. Janik, http://xxx.lanl.gov/abs/hepth/0703243 hep-th/0703243.

[65] R. J. Fries, J. I. Kapusta, and Y. Li, http://xxx.lanl.gov/abs/nuclth/0604054 nucl-th/0604054.

[66] D. Z. Fefferman and C. R. Graham, Conformal invariants, Elie Cartan et les Mathématiques d' aujourd' hui (Astérisque, 1985), p. 95.

[67] S. de Haro, S. N. Solodukhin, and K. Skenderis, Commun. Math. Phys. 217, 595 (2001).

[68] K. Fukushima, http://xxx.lanl.gov/abs/arXiv:0704.3625 [hepph] arXiv:0704.3625 [hep-ph].

[69] P. F. Kolb and U. W. Heinz, Invited Review for 'Quark Gluon Plasma 3', edited by R. Hwa and X. N. Wang (World Scientific, Singapore), p. 634. 